\title{
PONTE ET QUALITÉ D’OEUFS DE CAILLES ÉLEVÉES EN CONDITIONS SEMI INTENSIVES DANS L'EST ALGÉRIEN
}

\author{
LAYING AND EGGS QUALITY OF QUAILS IN SEMI-INTENSIVE CONDITIONS \\ INEASTERN ALGERIA
}

\begin{abstract}
Moula, N. ${ }^{1 *}$; Philippe, F.X. ${ }^{1}$; Ait Kaki, A. ${ }^{2}$; Touazi, L. ${ }^{3}$; Antoine-Moussiaux, N. ${ }^{1}$ et Leroy, P. ${ }^{1}$
${ }^{1}$ Faculté de Médecine Vétérinaire. Département des Productions animales. Service de Biostatistique, Bioinformatique, Economie, Sélection Animale.Université de Liège. Liège. Belgique.*Nassim.Moula@ulg.ac.be 2Université Constantine 1. Algérie.

${ }^{3}$ Université Ferhat Abbas. Sétif-1. Algérie.
\end{abstract}

\section{MOTS CLÉS ADDITIONNELS}

Coturnix coturnix japonica.

\section{RÉSUMÉ}

Le présent travail propose une évaluation des performances de ponte et de la qualité des œufs de cailles. Pour cette étude, les oeufs de 200 cailles ont été récoltés toutes les 4 semaines à partir de la $12^{2 \mathrm{ème}}$ semaine d'âge jusque la $44^{\text {ème }}$ semaine d'âge. Pour chaque période de récolte, les 240 premiers oeufs ont été pris en compte. Le taux de ponte moyen calculé sur une période de 37 semaines est de $74,44 \%$. Tous les paramètres de qualité d'oeufs étudiés ont été significativement $(p<0,001)$ influencés par l'âge des cailles. Les poids moyens de l'œuf entier (13,66 g), du jaune $(3,99 \mathrm{~g})$ et de la coquille $(2,28 \mathrm{~g})$ atteignent leurs valeurs maximales à la $40^{\text {ème }}$ semaine. Le poids moyen du blanc arrive à sa valeur maximale de $7,45 \mathrm{gr}$ à la $32^{\mathrm{ème}}$ semaine. Les indices du blanc $(12,02)$ et du jaune $(48,72)$ ont présenté leurs valeurs maximales respectivement à la $12^{\mathrm{ème}}$ et à la $16^{\text {ème }}$ semaine d'âge. La fraicheur des oeufs, exprimée par les unités d'Haugh diminue avec l'âge. Elle passe de 89,56 Unités d'Haugh (UH) à la $12^{\text {ème }}$ semaine d'âge à $83,71 \mathrm{UH}$ à la $44^{\text {ème }}$ semaine d'âge. La couleur du jaune présente des valeurs avec des fluctuations irrégulières $(7,12$ à 9,33$)$ selon une échelle colorimétrique de 15 échelles (l'éventail DSM de couleurs du jaune de l'oeuf). En conclusion, l'âge influence significativement les différents paramètres de qualité de l'oeuf de caille.

\section{SUMMARY}

This work investigates laying performances

\section{Additional KeYWORDS \\ Coturnix coturnix japonica.}

and egg quality of 200 quails during 266 laying days. The first collected 240 eggs, for the periods: $12,16,20,24,28,32,36,40,44$ weeks were used in this study. The average laying rate calculated over a period of 37 weeks is $74.44 \%$. All studied eggs quality parameters were significantly $(p<0.001)$ influenced by the age of the quail. The average weight of the whole egg (13.66 g), yolk $(3.99 \mathrm{~g})$ and shell $(2.28 \mathrm{~g})$ reach their maximum value at the $40^{\text {th }}$ week. The maximum albumen average weight $(7.45 \mathrm{~g})$ was observed at $32^{\text {nd }}$ week. Albumen (12.02) and yolk (48.72) indices recorded their highest values at the $12^{\text {th }}$ and the $16^{\text {th }}$ weeks, respectively. The eggs freshness, expressed here by Haugh units decreased with age. They range from 89.56 at the $12^{\text {th }}$ week to 83.71 at the $44^{\text {th }}$ week of age. Yolk color registered values with irregular fluctuations (7.12 to 9.33 ) in a colorimetric scale of 15 ladders. Highly significant and positive correlations $(p<0.001)$ and were recorded between the whole egg weight and the weight of albumen, yolk and shell. To conclude, the quail age affects significantly the various egg quality parameters.

\section{INTRODUCTION}

La caille est une espèce importante pour la production de viande et d'œufs, grâce à sa croissance rapide, sa maturité sexuelle précoce (début de la ponte à 6-8 semaines), 
Tableau I. Evolution des paramètres de qualité interne et externe d'oufs de cailles en fonction de l'âge (Moyennes moindres carrées \pm erreur standard). (Internal and external quality of quail eggs as influenced by age (Least square means \pm standard error)).

\begin{tabular}{|c|c|c|c|c|c|c|c|c|c|c|c|c|}
\hline & & & & & e (en s & semaine & es) & & & ESM & M & $\mathrm{R}^{2}$ \\
\hline & 12 & 16 & 20 & 24 & 28 & 32 & 36 & 40 & 44 & & & \\
\hline oids l'oeuf g & $12,28^{k}$ & 12,55 & $12,87^{\dagger}$ & $12,89^{e}$ & $12,99^{d}$ & & $13,29^{c}$ & $13,66^{a}$ & $13,05^{d}$ & 0,03 & 12,99 & 39,43 \\
\hline ndice blanc \% & $12,02^{a}$ & $10,63^{\text {de }}$ & $11,11^{b}$ & $10,82^{c}$ & $10,30^{j}$ & $10,50^{\text {ef }}$ & $f 10,74^{\text {cd }}$ & $10,47^{\dagger}$ & $10,81^{c}$ & 0,05 & 10,81 & 29,62 \\
\hline Poids blancg & $7,14^{i}$ & $7,18^{f}$ & $7,36^{d}$ & $7,41^{\mathrm{b}}$ & $7,39^{c}$ & $7,45^{a}$ & $7,38^{c}$ & $7,38^{c}$ & $7,29^{e}$ & 0,01 & 7,33 & 46,53 \\
\hline Blanc, $\%$ & $58,28^{a}$ & $57,20^{b}$ & $57,22^{b}$ & $57,47^{b}$ & $56,88^{c}$ & $55,49^{e}$ & $55,69^{\text {ed }}$ & $54,19^{f}$ & $55,97^{d}$ & 0,12 & 56,47 & 28,44 \\
\hline Unités Haugh & $89,56^{a}$ & $86,58^{c}$ & $87,70^{\mathrm{b}}$ & $86,74^{c}$ & $86,07^{d}$ & $85,05^{\mathrm{e}}$ & $84,62^{f}$ & $85,99^{d}$ & $83,71^{j}$ & 0,12 & 86,19 & 32,28 \\
\hline Indic & $48,43^{b}$ & $48,72^{a}$ & $47,03^{c}$ & 46,8 & $47,11^{c}$ & $46,03^{\dagger}$ & $46,36^{e}$ & $45,66^{j}$ & $46,79^{d}$ & 0,08 & & 40,58 \\
\hline Poids & $3,51^{\dagger}$ & $3,80^{e}$ & $3,82^{\mathrm{e}}$ & $3,93^{b c}$ & $3,95^{\mathrm{b}}$ & $3,97^{a}$ & $3,91^{c}$ & $3,99^{a}$ & $3,86^{d}$ & 0,01 & 3,86 & 46,52 \\
\hline Jaune & $28,61^{d}$ & $30,29^{a}$ & $29,70^{\mathrm{b}}$ & $30,45^{a}$ & $30,38^{a r}$ & $29,59^{b c}$ & c $29,44^{c}$ & $29,28^{c}$ & $29,58^{b c}$ & 0,07 & 29,71 & 19,49 \\
\hline Couleurjaune & $7,81^{\dagger}$ & $7,92^{\text {cde }}$ & $7,97^{\text {ef }}$ & $7,90^{\mathrm{cd}}$ & $7,89^{\text {def }}$ & $8,33^{a}$ & $7,99^{c}$ & $7,94^{\mathrm{cd}}$ & $7,12^{\mathrm{b}}$ & 0,04 & 7,99 & 10,39 \\
\hline Poids coquilleg & $1,61^{\text {de }}$ & $1,57^{\mathrm{e}}$ & $1,68^{d}$ & $1,56^{e}$ & $1,66^{d}$ & $2,03^{b}$ & $1,99^{b}$ & $2,28^{a}$ & $1,91^{c}$ & 0,03 & 1,81 & 46,52 \\
\hline Coquille \% & $13,11^{d}$ & $12,51^{\mathrm{e}}$ & $13,08^{d}$ & $12,08^{e}$ & $12,74^{\text {de }}$ & $14,93^{b}$ & $14,87^{\mathrm{bc}}$ & $16,53^{a}$ & $14,46^{c}$ & 0,17 & 13,82 & 21,13 \\
\hline Epais coquille 10 & & & & & & & & $18,94^{\mathrm{e}}$ & & 0,07 & & 34,23 \\
\hline Indice forme \% & $77,86^{a}$ & $77,76^{b}$ & $77,64^{c}$ & $77,67^{c}$ & $77,40^{d}$ & $77,15^{\dagger}$ & $77,30^{e}$ & $77,07^{j}$ & $77,45^{d}$ & 0,03 & 77,47 & 27,38 \\
\hline
\end{tabular}

Effet de l'âge $p<0,001$, pour toutes les variables. $M=$ Moyenne; $E S M=$ Erreur standard moyenne; $R^{2}=$ Coefficient de détermination (\%); Sur la même colonne, lettres différentes indiquent différence $(p<0,05)$.

son taux de production (180 à 300 oeufs par an) et sa courte période d'incubation. (Schwartz et Allen, 1981; Garwood et Diehl, 1987; Reddish et al., 2003).

Ce travail vise à étudier les performances de ponte et les caractéristiques qualitatives de l'œuf de caille en fonction de leur âge.

\section{MATÉRIELETMÉTHODES}

Cette étude a été réalisée entre Août 2012 et Juin 2013, dans le Département (wilaya) de Mila à environ 300 km à l'Est d'Alger. Le suivi de la ponte a concerné 200 cailles (Coturnix coturnix japonica) ${ }^{1}$, élevées au sol, qui n'ont pas été stimulées par la lumière pour provoquer le début de

\footnotetext{
${ }^{1}$ Composition de l'aliment: Maïs $53 \%$; Soja $30 \%$; Huile de soja $2 \%$; Son $10 \%$; Calcaire $1 ; \mathrm{CO}_{3} \mathrm{Ca}$ $3 \%$ CMV (Vitamine A 13500 UI/kg; Vitamine D3 3000 UI/kg; Vitamine E $25 \mathrm{mg} / \mathrm{kg}$; Sulfate de cuivre $15 \mathrm{mg} / \mathrm{kg}) 1 \%$.
}

ponte. Elles ont subi les conditions lumineuses automnales précédant la première ponte avant d'être transférées dans un local à 16 heures de lumière. Le bâtiment d'élevage était ventilé et les températures variaient de 25 à $35^{\circ} \mathrm{C}$ pour les températures maximales et de 14 à $28{ }^{\circ} \mathrm{C}$ pour les températures minimales.

Les œufs ont été récoltés de la semaine 12 à la semaine 44 avec un intervalle de 4 semaines entre les périodes d'analyses. Les 240 premiers oeufs par période ont été analysés. Les paramètres évalués sont décrits dans pour Moula et al. (2010) et Zita et al. (2013).

L'analyse statistique a été réalisée avec le logiciel SAS (2001). Le modèle linéaire généralisé (GLM) a été utilisé pour l'analyse de variance, afin de déterminer les différeces entre les âges; aussi, les corrélations entre caractères ont été étudiées. 


\section{RÉSULTATSETDISCUSSION}

L'âge moyen d'entrée en ponte a été de 42 jours. Le taux de ponte pour la période d'étude ( 7 à 44 semaines d'âge) est de 74,44 $\%$. Ce taux est supérieur à ceux obtenus par Schwartz et Allen (1981) et Garwood et Diehl (1987) qui ont rapporté que le taux de ponte annuel d'une caille pouvait varier de 49 à $68 \%$.

La mortalité totale enregistrée lors de la phase de ponte est de $15,50 \%$. Faute d'examens post mortem, les raisons de cette mortalité élevée ne peuvent être expliquées.

Dans la présente étude, tous les paramètres de qualité des œufs ont été significativement influencés $(\mathrm{p}<0,001)$ par l'âge de la caille (tableau I). Des résultats similaires ont été enregistrés par Zita et al. (2013). Cependant dans l'étude de ces derniers, l'âge n'a pas influencé significativement les indices du jaune et de forme. De nombreux auteurs (Nagarajan et al., 1991; Gonzalez, 1995; Altan et al., 1998; Danilov, 2000, Nazligul et al., 2001; Orhan et al., 2001) ont rapporté que le poids de l'œuf augmente avec l'âge. Dans notre étude le poids de l'oeuf augmente avec l'âge jusqu'à la $40^{\text {ème }}$ semaine où il atteint son poids maximum de 13,66g. Dans leur étude, Ri et al. (2005) ont enregistré une augmentation du poids de l'oeuf jusque la $32^{\text {ème }}$ semaine d'âge.

Les poids de l'albumen et du jaune présentaient une tendance à l'augmentation avec l'âge, confirmant ainsi les résultats de Nazligul et al. (2001). La proportion du blanc avait tendance à diminuer, ce qui correspond aux résultats rapportés par Fletcher et al. (1983). Le pourcentage du jaune quant à lui, avait présenté des fluctuations irrégulières avec l'âge ce qui correspond aux résultats rapportés par Zita et al. (2013) entre 9 et 49 semaines d'âge des cailles.

L'indice du blanc présentait des valeurs variables avec l'âge sans présenter de tendance particulière. Des résultats contradictoires sont trouvés dans la littérature.
Sachdev et al. (1989) avaient enregistré une diminution de l'indice du blanc avec l'âge alors que Nagarajan et al. (1991) et Orhan et al. (2001) avaient rapporté que cet indice du blanc augmentait avec l'âge.

La fraîcheur de l'oeuf est représentée dans cette étude par les unités d'Haugh. Ces dernières diminuent avec l'âge des cailles. Les mêmes observations ont été enregistrées par Nazligul et al.,(2001), Orhan et al. (2001) et Zita et al. (2013).

L'indice du jaune présentait une tendance à la diminution avec l'âge des cailles. Ce constat est concordant avec les résultats de Zita et al. (2013). Ils sont toutefois contradictoires avec ceux de Nagarajan et al (1991) et Gonzalez (1995) rapportant une augmentation de l'indice du jaune avec l'âge des cailles.

La couleur du jaune présentait des valeurs fluctuant irrégulièrement avec l'âge. Cette observation correspond aux résultats de Zita et al. (2013). Cependant ces derniers avaient obtenu des valeurs très basses à savoir 4,87 à comparer à 7,99 enregistrées dans la présente étude. Cette observation pourrait être due à la différence d'alimentation entre les deux études. En effet, c'est l'alimentation de la poule qui conditionne cette coloration (Nys, 2000; Blum et Sauveur, 1996; Naraharid et Rajinir, 1999), et plus particulièrement la présence de deux pigments caroténoïdes xanthophylles: la lutéine et la zéaxanthine (Zongo et al., 1997). La lutéine contribue pour $70 \%$ à la coloration du jaune, mais une vingtaine d'autres caroténoïdes sont aussi présents (Bourre, 2003).

À l'image des résultats obtenus par Orhan et al. (2001) et Gonzalez (1995), l'indice de forme dans la présente étude diminuait avec l'augmentation de l'âge des cailles, les oeufs les plus allongés étant pondu en début de période de ponte.

Nos résultats pour le poids et la proportion de la coquille sont concordants avec Zita et al. (2013) qui ont enregistré des fluctuations dans l'évolution de ces deux 
paramètres avec l'âge des cailles.

La présente étude a confirmé l'influence de l'âge sur la qualité de l'oeuf. D'autres études doivent être menées pour associer

\section{BIBLIOGRAPHIE}

Altan, O.; Oguz, I. and Akbas, Y. 1998. Effects of selection for high body weight and age of hen on egg characteristics in Japanese quail (Coturnix coturnix japonica). Turk J Vet Anim Sci, 22: 467-473.

Blum, J.C. and Sauveur, B. 1996. Caractéristiques et qualité de l'œuf de poule. Cah Nutr Diet, 31: 369-378.

Bourre, J.M. 2003. Effect of animal feeding and nutritional value on derived products consumed by humans: are lipids concerned nearly exclusively? OCL - Oleagineux, Corps Gras, Lipides, 10: 405-424.

Danilov, R.V. 2000. Effect of hens age on quality of hatching eggs and embryonic development proceeding of $21^{\text {st }}$ World's Poultry Congress. Montreal. Canada.

Fletcher, D.L.; Britton, W.M.; Pesti, G.M. and Savage S.I. 1983. The relationship of later flock age and egg weight on egg component yields and solid content. Poult Sci, 62: 1800-1805.

Garwood, A.A. and Diehl, R.C.J. 1987. Body volume and density of live Coturnix quail and associated genetic relationship. Poultry Sci, 66: 1268.

Gonzalez, M. 1995. Influence of age on physical traits of Japanese quail (Coturnix coturnix japonica) eggs. Ann Zootech, 44: 307-312.

Moula, N.; Antoine-Moussiaux, N.; Decuypere, E.; Farnir., F.; Mertens, K.; De Baerdemaeker, J. and Leroy P. 2010. Comparative study of egg quality traits in two Belgian local breeds and two commercial lines of chickens. Arch Geflügelk, 74: 164-171

Monira, K.N.; Salahuddin, M. and Miah, G. 2003. Effect of breed and holding period on egg quality characteristics of chicken. Int J Poult Sci, 2: 261-263.

Nagarajan, S.; Narhari, D.; Jayaprasad, I.A. and Thyagarajan, D. 1991. Influence of stocking density and layer age on production traits and egg quality in Japanese quail. British Poultry Sci, 32: 243-248. les autres facteurs tels que l'alimentation, la variété de la caille, le climat et le système d'élevage pour mieux cerner la qualité des oeufs de caille.

Naraharid, D. and Rajinir, R.A. 1999. Effect of dietary pearl millet and pigments on egg quality. Indian J Poult Sci, 34: 89-91.

Nazligul, A. ; Turkyilmaz, K. and Bardakcioglu, H.E. 2001. A study on some production traits and egg quality characteristics of Japanese quail. Turk $J$ Vet Anim Sci, 25: 1007-1013.

Nys, Y. 2000. Dietary carotenoids and egg yolk coloration: a review. Arch Geflugelk, 64: 45-54.

Orhan, H.; Erensaying, C. and Aktan, S. 2001. Determining egg quality characteristics of japanese quails (Coturnix Coturnix Japonica) at different ages. J Anim Produc, 42: 44-49.

Reddish, J.M.; Nestor, K.E. and Lilburn, M.S. 2003. Effect of selection for growth on onset of sexual maturity in randombred and growthselected lines of Japanese quail. Poultry Sci, 82: 187-191.

Ri, E.; Sato, K.; Oilkawa, T.; Kunieda, T. and Uchida, H. 2005. Effects of dietary protein levels on production and characteristics of Japanese quail eggs. J Poultry Sci, 42: 130-139.

Sachdev, A.K.; Ahuja, S.D. and Gopal, R. 1989. Feed consumption, egg production and egg quality traits as influenced by cage-tier locations of Japanese quail. Indian J Anim Sci, 59: 860865.

SAS. Statistical Analysis System. 2000. SAS/ STAT User's Guide. Version 8. SAS Inst. Inc. Cary. NC.

Schwartz, R.W. and Allen, N.K. 1981. Effect of aging on the protein requirement of mature female japanese quail for egg production. Poult Sci, 2: 342-348.

Zita, L.; Ledvinka, Z. and Klesalová, L. 2013. The effect of the age of Japanese quails on certain egg quality traits and their relationships. Vet Arch, 83: 223-232.

Zongo, D.; Ba, C.; Diambra, O. and Coulibaly M. 1997. Coloration effect of a natural source of pigment (Leucaena leucocephala) for use in poultry. Ann Zootech, 46 : 185-190. 